\title{
Selective Medium for Isolation of Ureaplasmas from Animals
}

\author{
Kaoru KOSHIMIZU, Masahiro ITO, Teruo MAGARIBUCHI, and Hitoshi KOTANI ${ }^{1)}$ \\ Division of Animal Research, Faculty of Medicine, University of Tokyo, Bunkyo, Tokyo 113, \\ and 1'Imamichi Institute for Antmal Reproduction, Niihari, Ibaraki, 300-01
}

(Received 1 October 1982/Accepted 18 January 1983)

Since the first isolation of ureaplasmas from human urogenital tracts by Shepard [22] in 1954, these organisms have been found to be harbored in several species of primates $[2,4,14,18,27]$, carnivora $[3,8,11,13,26,27]$, artiodactyla $[5-7,15,16,20,25,28]$, proboscidae [29], and aves $[9,10,24]$. However, in spite of our intensive studies [12], ureaplasmas have not been isolated from other animal species.

There have been some difficulties in isolating ureaplasmas from animals. A major problem is that the overgrowth of so-called large colony mycoplasmas on isolation media has frequently made isolation of ureaplasmas difficult. Therefore, it seems to be of the uppermost importance to make a selective medium for isolation of ureaplasmas from animals.

Shipley and Bowman [23] first reported in 1968 that human ureaplasmas were resistant to lincomycin. Since then this agent has been added to the medium for recovery and purification of human ureaplasmas from mixed cultures containing both Ureaplasma urealyticum and Mycoplasma hominis [1, 19, 21], because the growth of the latter is inhibited by lincomycin. Nagatomo and Shimizu [17] reported that minimum inhibitory concentrations (MIC) of lincomycin for 11 strains of bovine ureaplasmas were 50 to
$100 \mu \mathrm{g}$ per $\mathrm{m} l$. However, there has been no report on the sensitivities to lincomycin of ureaplasmas from other animals. In the present study, the MICs of lincomycin for various strains and species of ureaplasmas, mycoplasmas and acholeplasmas were compared, and a suitable concentration of lincomycin for selective isolation of animal ureaplasmas was determined. The strains of these organisms are listed in Table 1. The liquid medium used in the test was $T$-broth described previously [10]. The MICs of lincomycin were defined by the lowest concentrations of the drug preventing color change of the medium in microtiter wells containing $10^{3}$ to $10^{4}$ color changing unit per $\mathrm{ml}$ of the test organisms, when the $\mathrm{pH}$ of the control wells containing organisms in the medium without the drug reached 7.8.

It was disclosed that ureaplasmas of animal origin were resistant to lincomycin as shown in Table 1 , and 12.5 to 100 $\mu \mathrm{g}$ per $\mathrm{m} l$ of the drug were the minimum inhibitory concentrations for human, bovine, simian, canine, feline and avian ureaplasmas. On the contrary, MICs of lincomycin for various species of mycoplasmas and acholeplasmas were between 0.2 and $3.13 \mu \mathrm{g}$ per $\mathrm{ml}$, except those for $M$. capricolum California kid, $M$. gallisepticum PG31, and M. gallisepticum S6 being 25.0, 25.0 and 12.5 $\mu \mathrm{g}$ per $\mathrm{ml}$, 
Table 1. Sensitivities of various mycoplasma, acholeplasma and ureaplasma strains to lincomycin

\begin{tabular}{|c|c|c|c|c|c|c|c|c|c|c|c|}
\hline \multicolumn{12}{|c|}{ Minimum inhibitory concentration $(\mu \mathrm{g} / \mathrm{m} l)$} \\
\hline \multirow[t]{6}{*}{0.05} & 0.1 & 0.20 & 0.39 & 0.78 & 1.56 & 3.13 & 6.25 & 12.5 & 25 & 50 & 100 \\
\hline & & (2) (21) & (3) (5) & (1) (4) & (6) (14) & (7) (8) & & (28) 39 & 9 (11) (27) & 4246 & 4143 \\
\hline & & 37 & (15) (25) & (9) (19) & (18) (20) & (10) (12) & & & 3840 & 5051 & 4453 \\
\hline & & & 3033 & & (22) 32 & (13) (16) & & & 4547 & 54 & 55 \\
\hline & & & & & & (17) 23 & & & 4849 & & \\
\hline & & & & & & $\begin{array}{l}\text { (24) (26) } \\
\text { (29) } 36\end{array}$ & & & 52 & & \\
\hline \multicolumn{4}{|c|}{ (1) M. hominis PG21 } & \multicolumn{4}{|c|}{ (19) M. hyorhinis PG29 } & $38 \quad$ & \multicolumn{3}{|c|}{3 U. urealyticum No. 7} \\
\hline \multicolumn{4}{|c|}{ (2) M. buccale $\mathrm{CH} 20247$} & 20 & \multicolumn{3}{|c|}{ M. arthritidis PG6 } & $39 L$ & \multicolumn{3}{|c|}{ U. urealyticum No. 23} \\
\hline \multicolumn{4}{|c|}{ (3) M. fermentans PG 18} & (21) & \multicolumn{3}{|c|}{ M. caviae G122 } & $40 \quad L$ & \multicolumn{3}{|c|}{ U. urealyticum No. 27} \\
\hline \multicolumn{4}{|c|}{ (4) $M$. salivarium PG20 } & (22) & \multicolumn{3}{|c|}{ M. neurolyticum PG28 } & $41 \quad L$ & \multicolumn{3}{|c|}{ U. urealyticum No. 58} \\
\hline \multicolumn{4}{|c|}{ (5) $M$. orale CH19299 } & 23 & \multicolumn{3}{|c|}{ M. pulmonis PG22 } & $42 \quad L$ & \multicolumn{3}{|c|}{ U. urealyticum No. 354} \\
\hline \multicolumn{4}{|c|}{ (6) M. alkalescens D12 } & (24) & \multicolumn{3}{|c|}{ M. pulmonis m53 } & $43 \quad L$ & \multicolumn{3}{|c|}{ U. urealyticum Pirillo } \\
\hline \multicolumn{4}{|c|}{ (7) M. bovngenitalium PG11 } & (25) & \multicolumn{3}{|c|}{ M. primatum HRC292 } & $44 \quad L$ & \multicolumn{3}{|c|}{ U. urealyticum Cook } \\
\hline \multicolumn{4}{|c|}{ (8) M. mycoides subsp. } & (26) & \multicolumn{3}{|c|}{ M. gallinarum PG16 } & $45 \quad L$ & \multicolumn{3}{|c|}{ U. urealyticum $\mathrm{T}-960$} \\
\hline \multicolumn{4}{|c|}{ mycoides $\mathrm{PGl}$} & (27) & \multicolumn{3}{|c|}{ M. gallisepticum PG31 } & $46 \mathrm{~B}$ & \multicolumn{3}{|c|}{ Bovine ureaplasma C2 } \\
\hline \multicolumn{4}{|c|}{ (9) M. agalactiae PG2 } & 28 & M. gallise & epticum S6 & & $47 \mathrm{~B}$ & Bovine ure: & aplasma $\mathrm{C}$ & $\mathrm{C} 13$ \\
\hline (10) $N$ & M. argin & ni G230 & & (29) & $M$. iners & PG30 & & $48 \mathrm{~B}$ & Bovine ure: & aplasma $A$ & A417 \\
\hline (11) $N$ & M. capri & lum Calif & ornia kid & 30 & A. modicu & im PG49 & & $49 \mathrm{C}$ & Canine ure & aplasma I & $\mathrm{D} 6 \mathrm{P}-\mathrm{C}$ \\
\hline (12) $N$ & M. canis & G14 & & $3 \mathrm{~B}$ & A. laidlaz & wii PG10 & & $50 \mathrm{C}$ & Canine ure & aplasma 1 & DIM-C \\
\hline (13) $N$ & M. edwa & tii PG24 & & 32 & A. laidlaz & wii PG8 & & $51 \mathrm{~F}$ & Feline urea & plasma $\mathbf{F}$ & $45 \mathrm{~d} 2$ \\
\hline & M. macu & sum PG1 & & 33 & A. equifer & tale C112 & & $52 \mathrm{~F}$ & Feline urea & plasma $\mathrm{F}$ & \\
\hline (15) $N$ & M. mora & $\mathrm{H} 542$ & & 34 & A. hippik & on $\mathrm{Cl}$ & & $53 \mathrm{~S}$ & Simian urea & aplasma I & Т $167-1$ \\
\hline (16) $\Lambda$ & M. spum & ns PG 13 & & 35 & A. granu & larum BTS3 & & $54 \mathrm{~S}$ & Simian urea & aplasma T & T 163 \\
\hline (17) $N$ & M. felis & & & 36 & A. oculi & & & $55 A$ & Avian urea & plasma D & \\
\hline (18) $\Lambda$ & M. gatea & & & $\hat{3 \lambda}$ & A. axanth & ium S743 & & & & & \\
\hline
\end{tabular}

Table 2. Growth of ureaplasma strains on solid medium added with lincomycin

\begin{tabular}{|c|c|c|c|c|c|}
\hline \multirow{2}{*}{ Ureaplasma strain } & \multirow{2}{*}{$\begin{array}{c}\text { Dilution } \\
\text { of } \\
\text { culture }\end{array}$} & \multicolumn{4}{|c|}{ Concentration of lincomycin $(\mu \mathrm{g} / \mathrm{m} l)$} \\
\hline & & 0 & 5 & 10 & 20 \\
\hline \multirow{4}{*}{ Avian ureaplasma D6-1 } & $\Gamma 0$ & + & + & + & + \\
\hline & $10^{-1}$ & + & + & + & + \\
\hline & $10^{-2}$ & + & + & + & + \\
\hline & $\mathrm{L}_{10^{-3}}$ & + & + & + & $(+)$ \\
\hline \multirow{4}{*}{ Canine ureaplasma D $1 \mathrm{M}-\mathrm{C}$} & $\Gamma_{0}^{0}$ & + & + & + & - \\
\hline & $10^{-1}$ & + & + & $(+)$ & - \\
\hline & $10^{-2}$ & + & $(+)$ & - & - \\
\hline & $\mathrm{L}_{10^{-3}}$ & + & - & - & - \\
\hline \multirow{4}{*}{ Feline ureaplasma $\mathrm{F} 45 \mathrm{~d} 2$} & $\Gamma_{0}^{0}$ & + & + & $(+)$ & - \\
\hline & $10^{-1}$ & + & + & - & - \\
\hline & $10^{-2}$ & + & - & - & - \\
\hline & $\mathrm{L}_{10^{-3}}$ & + & - & - & - \\
\hline
\end{tabular}

+; marked growth of colony, (+); faint growth of colony, - ; no growh of colony. 
respectively.

As shown in Table 2, avian ureaplasmas grew on solid media containing 5 to $20 \mu \mathrm{g}$ per $\mathrm{m} l$ of lincomycin. Although canine and feline ureaplasmas were found to be somewhat sensitive to linco-

Table 3. Selective media for isolation of ureaplasmas from animals

\section{Liquid medium:}

PPLO broth w/o CV (Difco)

Horse serum

$25 \%(\mathrm{w} / \mathrm{v})$ yeast extract (Nitten Co.) $5 \mathrm{ml}$

100,000 units of penicillin $\mathrm{G} \quad 1 \mathrm{ml}$

$2.5 \%$ thallium acetate $\quad 1 \mathrm{ml}$

$500 \mu \mathrm{g}$ of lincomycin $\quad 1 \mathrm{ml}$

$10 \%$ urea

$0.2 \%$ phenol red

$\mathrm{pH}$

$1 \mathrm{ml}$

$1 \mathrm{ml}$

6.2

Solid medium:

Liquid medium

$100 \mathrm{ml}$

Phosphate buffer

(7:3 mixture of $1.5 \mathrm{M} \mathrm{KH}_{2} \mathrm{PO}_{4}$ and

$\left.1.5 \mathrm{M} \mathrm{Na}_{2} \mathrm{HPO}_{4}\right)$

Purified agar (Oxoid)

$\mathrm{pH}$ mycin at $5 \mu \mathrm{g}$ per $\mathrm{m} l$, addition of $5 \mu \mathrm{g}$ per $\mathrm{m} l$ of the drug to liquid and solid media was adopted for selective isolation of ureaplasmas from animal specimens.

The ingredients of the improved solid and liquid media for selective isolation of ureaplasmas from animals are shown in Table 3. The solid medium consisted of the same ingredients as the liquid one, except that $2.5 \mathrm{~m} l$ of phosphate buffer, which was reported by Windsor [30] in 1975 , and $0.8 \mathrm{~g}$ of purified agar (Oxoid) were added, with the $\mathrm{pH}$ adjusted to 6.5 .

Using both direct plating and enrichment methods, we compared isolation of ureaplasmas from oral cavities of several species of animals. The isolation methods were described previously [10].

Fig. 1 shows comparative results of isolation of ureaplasmas from canine oral cavities with media with and without lincomycin. In both direct plating and enrichment methods, ureaplasmas were more easily isolated in media containing

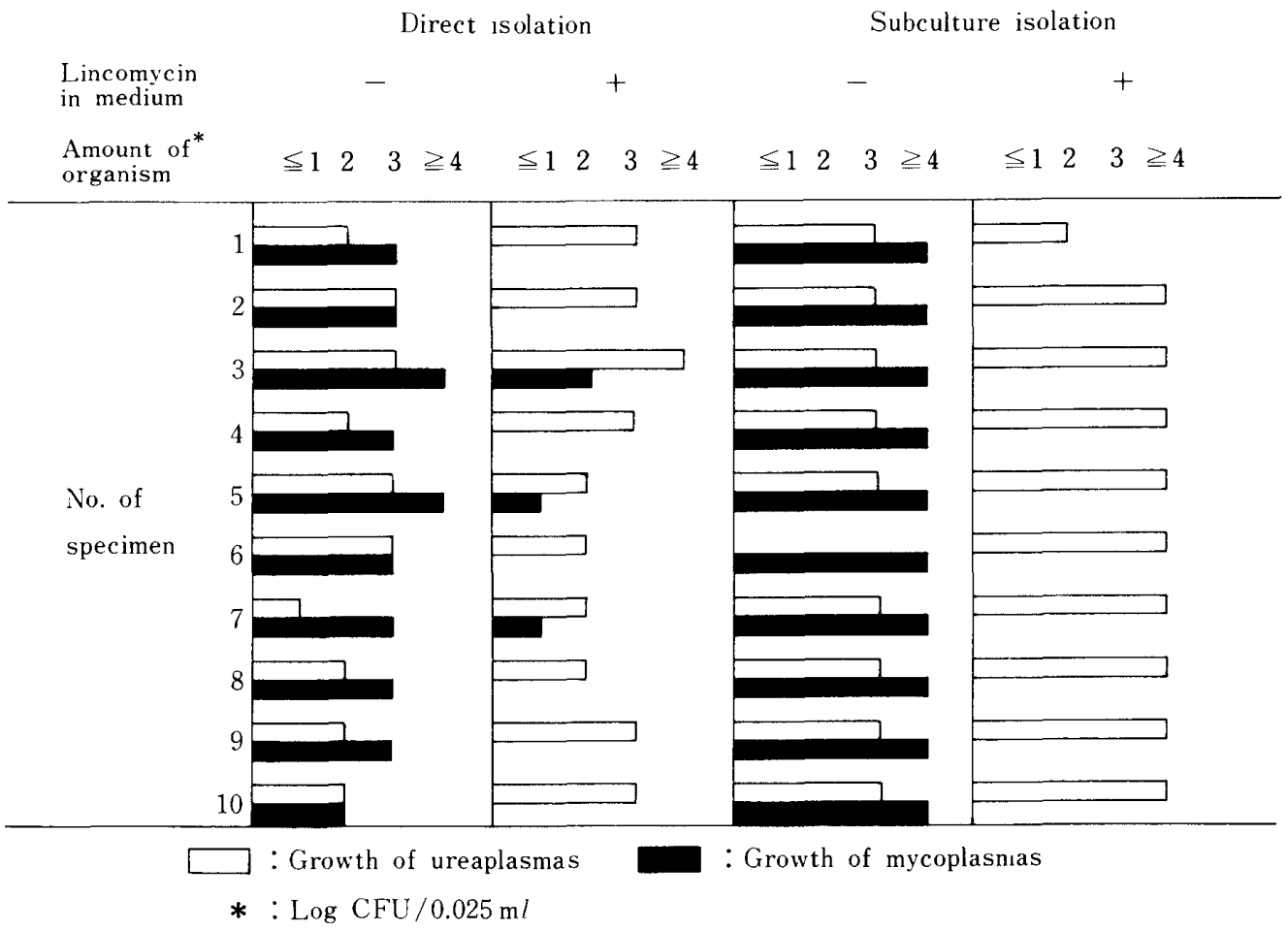

Fig. 1. Selective isolation of ureaplasmas from canine oral cavities in media with and without lincomycin. 
lincomycin than in lincomycin-free ones.

Fig. 2 shows mixed colonies of ureaplasmas and mycoplasmas having developed on the solid medium without lincomycin, directly inoculated with a canine oral swab specimen. Purification of an ureaplasma colony from this culture was very difficult. In contrast with Fig. 2, Fig. 3 shows ureaplasma colonies alone having developed on the solid medium with lincomycin inoculated with the same specimen. No mycoplasma colony developed on this medium, and it was easy to isolate ureaplasma colonies for further cloning. Furthermore, similar results were obtained in isolation of ureaplasmas from bovine, feline, and chicken oral cavities.

This study clarified that isolation media containing $5 \mu \mathrm{g}$ per $\mathrm{m} l$ of lincomycin were very selective for isolation of ureaplasmas from animals. Therefore, these media will provide a useful tool for the survey of ureaplasmas in nature.

ACknowledgments. The authors thank Dr. I. Z. Pan of Department of Veterinary Microbiology, Faculty of Agriculture, University of Tokyo, for his technical assistances.

\section{REFERENCES}

1. Braun, P., Klein, J. O., and Kass, E. H. (1970). App. Microbiol, 19, 62-70.

2. Brown, W. J., Jacob, N. F., Jr., Arum, E. S., and Arko, R. J. (1976). Lab. Anim. Sci. 26, 81-83.

3. Friis, N. F., and Pedersen, K. B. (1980). Acta, Vet. Scand. 21, 134-136.

4. Furr, P. M., Taylor-Robinson, D., Hetherington, C. M. (1976). Lab. Anim. 10, 393-398.

5. Doig, P. A. (1977). Vet. Rec. 100, 179-180.

6. Gourlay, R. N. (1968). Res. Vet. Sci. 9, 367377.

7. Gourlay, R. N., Brownlie, J., and Howard, C. J. (1973). J. Gen. Microbiol. 76, 25 1-254.

8. Harasawa, R., Yamamoto, K., and Ogata, M. (1977). Microbiol. Immunol. 21, 179-181.

9. Koshimizu, K., and Magaribuchi, T. (1977).
Jpn. J. Vet. Sci. 39, 195-199.

10. Koshimizu, K., and Magaribuchi, T. (1978). Jpn. J. Vet. Sci. 40, 719-727.

11. Koshimizu, K., Magaribuchi, T., and Ito, M. (1979). Jpn. J. Vet. Sci. 41, 545-549.

12. Koshimizu, K., Magaribuchi, T., Ito, M., Kotani, H., and Ogata, M. (1981). Vet. Rec. 108, $309-310$

13. Kotani, H., and Ogata, M. (1979). Jpn. J. Vet. Sci. 41, 639-646.

14. Kundsin, R. B., Rowell, T., Shepard, M. C., Parreno, A., and Lanceford, C. D. (1975). Lab. Anim. Sci. 25, 221-224.

15. Livingston, C. W., Jr., and Gauer, B. B. (1975). Amer. J. Vet. Res. 36, 313-314.

16. Livingston, C. W., Jr. (1972). Amer. J. Vet. Res. 33, 1925-1929.

17. Nagatomo, H., and Shimizu, T. (1976). J. Jpn. Vet. Med. Assoc. 29, 273-276.

18. Ogata, M., Kotani, H., Koshimizu, K., and Magaribuchi, T. (1981). Jpn. J. Vet. Sci. 43, $521-529$.

19. Robertson, J. A. (1978). J. Clin. Microbiol. 7, $127-132$.

20. Ruhnke, H. L., and Van Dreumel, A. A. (1971). Canad. J. Comp. Med. Vet. Sci. 36, 317-318.

21. Shepard, M. C. (1974). Collog. Inst. Natl Sante Rech. Med. (INSERM). 33, 375-380.

22. Shepard, M. C. (1954). Amer. J. Syph. Gonor. Vener. Dis. 38, 113-124.

23. Shipley, A., and Bowman, S. J. (1968). Med. J. Aust. 1, 794-796.

24. Stipkovits, L., and Rashwan, A. (1976). Proc. Soc. Gen. Microbiol. 13, 158.

25. Stipkovits, L., Rashwan, A., Takcs, J., and Lapis, K. (1978). Zentralbl. Veterinaermed. B, 25, 605-608.

26. Tan, R. J. S., and Markham, J. G. (1971). Jap. J. Exp. Med. 41, 247-248.

27. Taylor-Robinson, D., Martin-Bourgon, C., Watanabe, T., and Addy, J. P. (1971). J. Gen. Microbiol. 68, 97-107.

28. Taylor-Robinson, D., Haig, D. H., and Williams, M. H. (1967). Ann. N. Y. Acad. Sci. $143,517-518$.

29. Taylor-Robinson, and Furr, unpublished data (1980). In I. O. M. International Research Programme on Comparative Mycoplasmology, Report of Consultations, Custer, U.S.A., 13 .

30. Windsor, G. D., Edward, D. G. ff., and Trigwell, J. A. (1975). J. Med. Microbiol., 183187. 


\section{EXPLANATION OF FiguRES}

Fig. 2. Mycoplasma (arrow a) and ureaplasma (arrow b) colonies on solid medium without lincomycin inoculated with a canine oral specimen.
Fig. 3. Ureaplasma colonies alone developed on solid medium with $5 \mu \mathrm{g}$ of lincomycin per $\mathrm{m} l$ inoculated with the same specimen as mentioned in Fig. 2.

要約

動物からのウレアプラズマ選択分離培地 (短報)：輿水 馨・伊藤正博・曲㴊輝夫・小谷 均 ${ }^{1)}$ (東 京大学医学部動物実験施設, 1) 動物繁殖研究所)——動物からのウレアプラズマ選択分離培地につい て検討した。ヒト抢よび各種動物由来ウレアプラズマ 18 株に対するリンコマイシンの最小発有阻止 濃度 $(\mathrm{MIC})$ は $12.5 \sim 100 \mu \mathrm{g} / \mathrm{ml}$ であり, 一方，各種動物由来マイコプラズマ, アコレプラズマ計 37 株に対する本剂の MIC は大部分の株が $0.2 \sim 3.13 \mu \mathrm{g} / \mathrm{ml}$ を示した。常用分離培地にリンコマイシン $5 \mu \mathrm{g} / \mathrm{ml}$ を添加したところ，マイコプラズマ抢よびアコレプラズマの増殖が抑制され，ウレアプラズ マが選択的に分離された。 

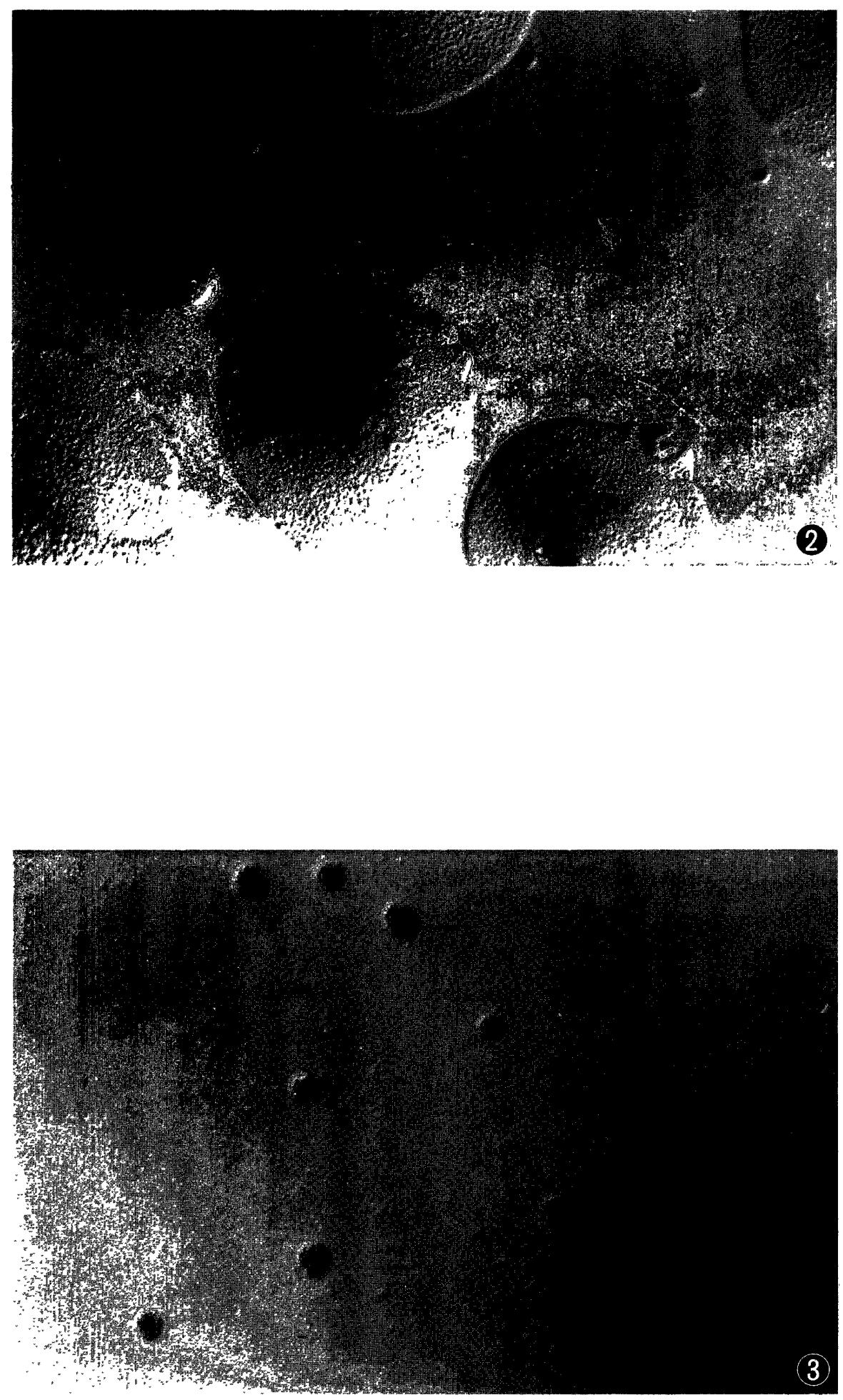